\title{
Quasi-Brittle Fracture Modeling of Preflawed Bitumen Using a Diffuse Interface Model
}

\author{
Yue Hou, ${ }^{1}$ Fengyan Sun, ${ }^{1}$ Wenjuan Sun, ${ }^{2}$ Meng Guo, ${ }^{1}$ Chao Xing, ${ }^{3}$ and Jiangfeng Wu ${ }^{1}$ \\ ${ }^{1}$ National Center for Materials Service Safety, University of Science and Technology Beijing, Beijing 100083, China \\ ${ }^{2}$ The Via Department of Civil and Environmental Engineering, Virginia Polytechnic Institute and State University, \\ Blacksburg, VA 24061, USA \\ ${ }^{3}$ School of Transportation Science and Engineering, Harbin Institute of Technology, Harbin 150001, China
}

Correspondence should be addressed to Fengyan Sun; fysun@ustb.edu.cn

Received 21 March 2016; Accepted 11 April 2016

Academic Editor: Liviu Marsavina

Copyright (C) 2016 Yue Hou et al. This is an open access article distributed under the Creative Commons Attribution License, which permits unrestricted use, distribution, and reproduction in any medium, provided the original work is properly cited.

Fundamental understandings on the bitumen fracture mechanism are vital to improve the mixture design of asphalt concrete. In this paper, a diffuse interface model, namely, phase-field method is used for modeling the quasi-brittle fracture in bitumen. This method describes the microstructure using a phase-field variable which assumes one in the intact solid and negative one in the crack region. Only the elastic energy will directly contribute to cracking. To account for the growth of cracks, a nonconserved Allen-Cahn equation is adopted to evolve the phase-field variable. Numerical simulations of fracture are performed in bituminous materials with the consideration of quasi-brittle properties. It is found that the simulation results agree well with classic fracture mechanics.

\section{Introduction}

One of the most serious distresses in pavement structure is brittle cracking in bitumen [1,2]. The Strategic Highway Research Program (SHRP) realized the importance of the cracking properties of bitumen for the first time [3]. Ayatollahi and Pirmohammad (2013) studied the temperature effects on brittle fracture in cracked asphalt concrete [4]. Rowe et al. (2014) conducted researches on the influence of binder rheology on the cracking of asphalt mixes in airport and highway projects [5]. Dave et al. (2007) studied the thermal reflective cracking of asphalt concrete overlays [6]. Birgisson et al. (2002) predicted quasi-brittle response and crack growth in asphalt mixture with the Boundary Element Method [7]. Ameri et al. (2011) calculated the stress intensity factor in cracked asphalt pavement under traffic loading using 3D finite element analysis [8]. Tan and Guo (2014) studied the interfacial interaction between asphalt and mineral fillers and the influence on asphalt cracking [9]. Hou et al. (2014) conducted researches on the mixed mode fracture failure of asphalt binder at low temperature using the phasefield approach [10]. Feng et al. (2007) studied the anticracking performance of the ATB30 asphalt treated base [11]. Mun and Lee (2011) modeled quasi-brittle crack growth in hotmix asphaltic concrete mixtures using a disk-shaped compact tension test [12]. Zhao and Zhang (2010) conducted research on low temperature cracking of asphalt pavement based on Cohesive Zone Model [13]. Almost all these analyses are based on classic fracture mechanics first proposed by Griffith [14], which needs to clearly depict the crack front conditions, and thus may be very complicated. In order to consider the quasi-brittle properties of bitumen concisely, phase-field method is used in this paper. Besides, although the authors have previously analyzed the asphalt cracking using phasefield method [15], there still have been two limits: (1) the previous research is focused on the asphalt linear behavior and does not consider the quasi-brittle behavior; (2) there lacks analytical solutions on the stress during cracking.

The phase-field method (PFM) was originally proposed by Cahn and Hilliard [16]. In this model, a phase-field variable is introduced to identify different phases. In this paper, the development of an Allen-Cahn phase-field model is implemented in the finite element software COMSOL. The model was validated using the two-dimensional simulations 

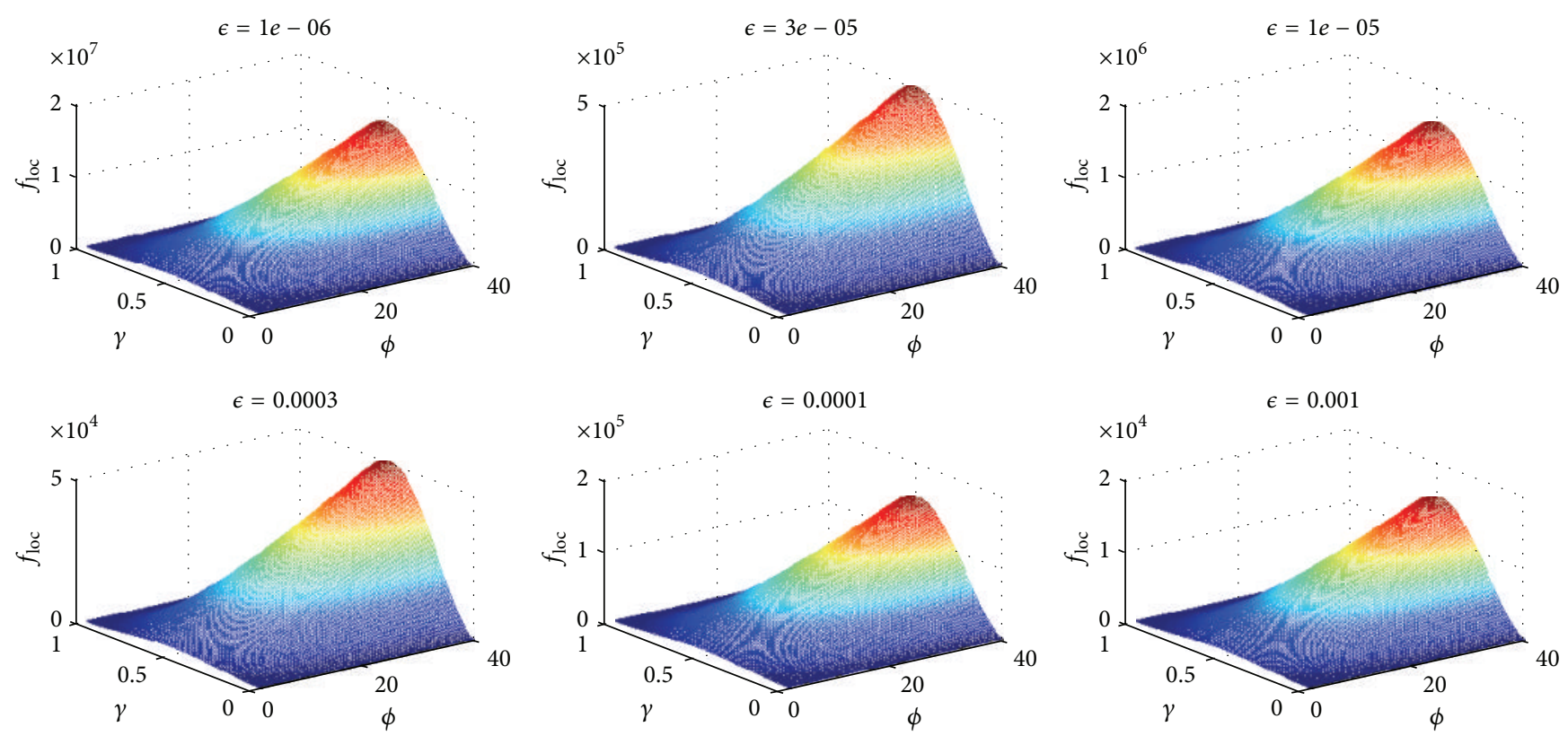

FIGURE 1: Local free energy density with respect to different $\epsilon$.

of model I quasi-brittle fracture at one fixed temperature and comparison with Griffith's theory.

\section{Theoretical Analysis}

2.1. Free Energy in Quasi-Brittle Materials. In the simulation of preflawed bitumen cracking, the phase-field variable is set as $\phi=-1$ for the broken part and $\phi=+1$ for the unbroken part. The total free energy in the cracking system is

$$
F=\int_{\Omega}\left(f_{\mathrm{gr}}+f_{\mathrm{loc}}+f_{\mathrm{el}}\right) d V
$$

where $f_{\text {el }}$ is the elastic energy density, $f_{\mathrm{gr}}=3 \gamma \epsilon(\nabla \phi)^{2} / 2$ is the gradient energy density, and $f_{\text {loc }}=6 \gamma \phi^{2}(1-\phi)^{2} / \epsilon$ is the local free energy density (double well potential). The rationale of free energy composition in asphalt material is well explained in Hou et al. (2014) [10]. $\gamma$ is the surface free energy; and $\epsilon$ the interface coefficient.

Figure 1 shows the double well potential function with respect to different $\epsilon$. It is observed that the smaller the interface coefficient $\epsilon$ is, the larger the peak value of $f_{\text {loc }}$ is. Also, note that curvature is significantly larger for a large surface free energy $\gamma$, which indicates that bitumen has more cracking resistance for a large $\gamma$.

Figure 2 shows the double well potential function with respect to different $\gamma$. It is observed that the larger the surface free energy $\gamma$ is, the larger the peak value of $f_{\text {loc }}$ is. Also note for a fixed $\gamma$ value, there exist two minima at $\phi=0$ and $\phi=1$, which indicates only these two states are metastable.

Figure 3 shows the double well potential function with respect to different $\phi$. Note that there are three important states: $\phi=0$ represents the cracking state, $\phi=0.5$ represents the interface state, and $\phi=1$ represents the unbroken state.
For these three states, the system is metastable; that is, $f_{\text {loc }}=$ 0 . Other than these states, the system is unstable.

Actually, for bitumen and bituminous materials, the materials are quasi-brittle since the visco- and plastic properties cannot be neglected no matter how small their magnitudes are. The cracking process is always initiated by the local plastic deformation at the crack tip. The plastic work needs to be done first before crack can advance to create new surfaces. The total stress is thus considered as the sum of the elastic part, the viscous part and, the plastic part as

$$
\sigma_{i k}^{\mathrm{total}}=\sigma_{i k}^{\mathrm{el}}\left(\varepsilon_{i k}\right)+\sigma_{i k}^{\mathrm{vis}}\left(\dot{\varepsilon}_{i k}\right)+\sigma_{i k}^{\mathrm{pl}} .
$$

For low temperature cracking on bitumen and bituminous materials, the latter two terms in (2) can be neglected for approximation.

The elastic part can be obtained according to Hooke's law as

$$
\sigma_{i k}^{\mathrm{el}}=\frac{E}{1+\nu}\left(\varepsilon_{i k}+\frac{1}{1-2 \nu} \delta_{i k} \varepsilon_{i i}\right)
$$

where $\delta_{i k}$ is Kronecker delta.

The viscous part could similarly be obtained as [17]

$$
\sigma_{i k}^{\mathrm{vis}}=\frac{\eta}{1+\zeta}\left(\dot{\varepsilon}_{i k}+\frac{1}{1-2 \zeta} \delta_{i k} \dot{\varepsilon}_{l l}\right),
$$

where $\eta$ and $\zeta$ are two viscosity constants defined similar to the elastic constants in (3).

The plastic stress is obtained as

$$
\sigma_{i k}^{\mathrm{pl}}=\sqrt{\frac{1}{2}\left[\left(\sigma_{x}-\sigma_{y}\right)^{2}+\left(\sigma_{x}-\sigma_{z}\right)^{2}+\left(\sigma_{y}-\sigma_{z}\right)^{2}\right]},
$$

where $\sigma_{x}, \sigma_{y}$, and $\sigma_{z}$ are the localized stress. 

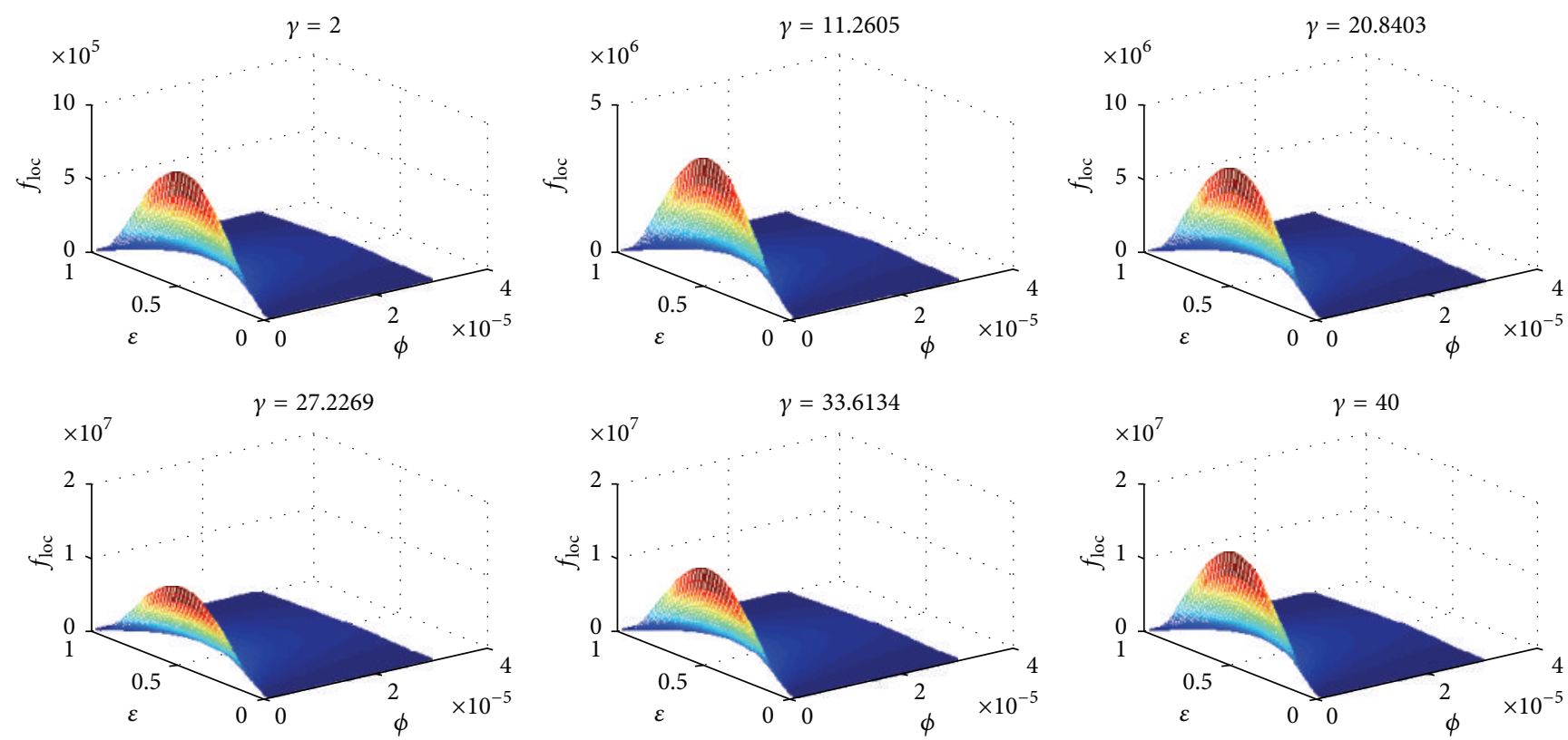

FIGURE 2: Local free energy density with respect to different $\gamma$.
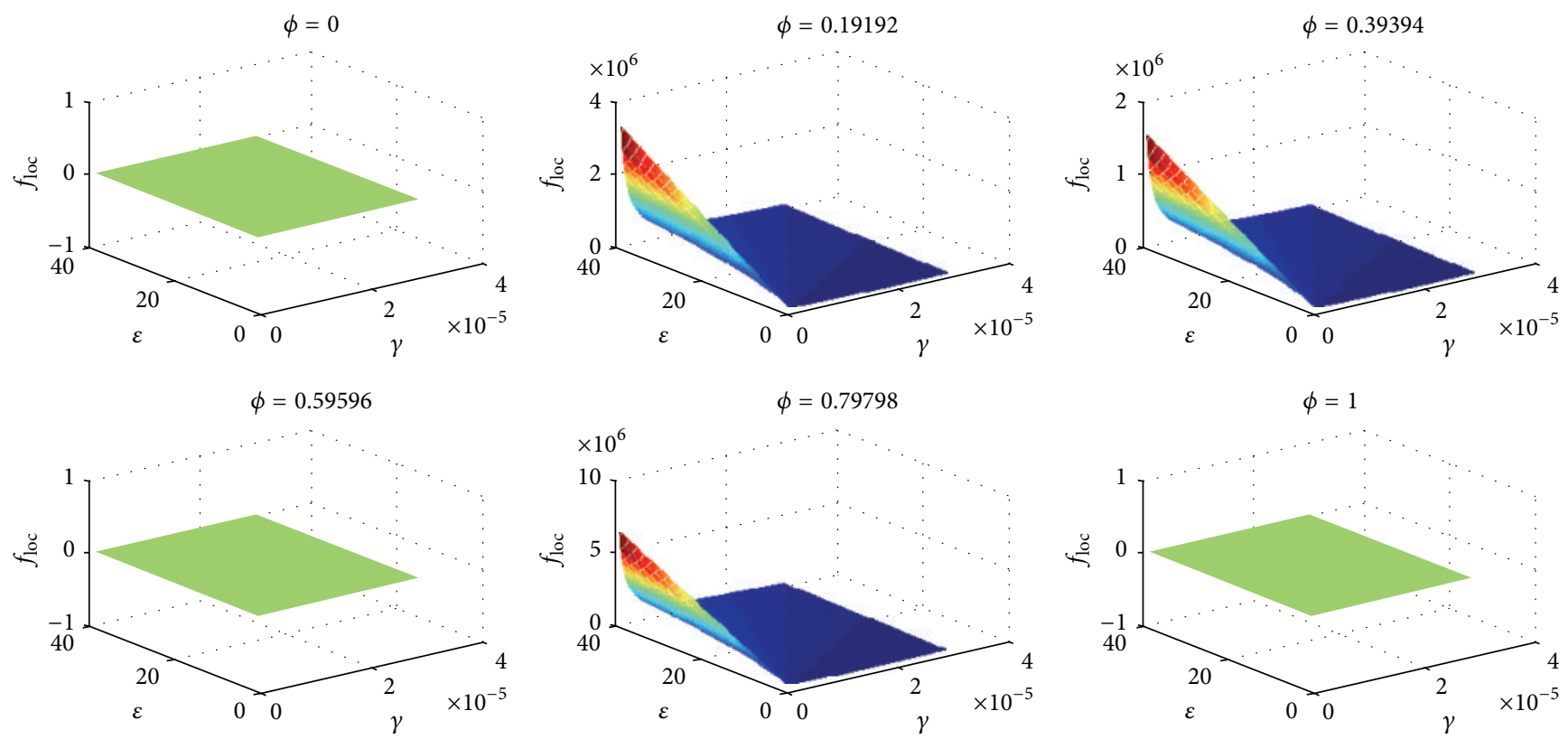

FIGURE 3: Local free energy density with respect to different $\phi$.

2.2. Momentum Equations. The evolution of the stress fields is determined by the principle of momentum conservation:

$$
\rho \ddot{u}_{i}=\frac{\partial}{\partial x_{i}}\left(\sigma_{i k}^{\mathrm{el}}+\sigma_{i k}^{\mathrm{vis}}+\sigma_{i k}^{\mathrm{pl}}\right) .
$$

In order to implement phase-field in the stress field, the elastic modulus should be modified as

$$
\sigma_{i k}=\frac{E(\phi)}{1+\nu}\left(\varepsilon_{i k}+\frac{1}{1-2 \nu} \delta_{i k} \varepsilon_{i i}\right), \quad \text { (7) } \quad \frac{\partial \phi}{\partial t}=-\frac{D}{3 \gamma \epsilon} \psi
$$

where $E(\phi)$ is the elastic modulus and $v$ is Poisson's ratio. And $E(\phi)=E+\left(E-E_{0}\right)\left(-(1 / 4) \phi^{3}+(3 / 4) \phi+(1 / 2)\right)$, where $E$ and $E_{0}$ are the elastic moduli of the bitumen and the broken part, respectively.

Note that the viscous part and plastic part almost have no contributions to quasi-brittle cracking. Allen-Cahn equation is employed as the governing equation: 


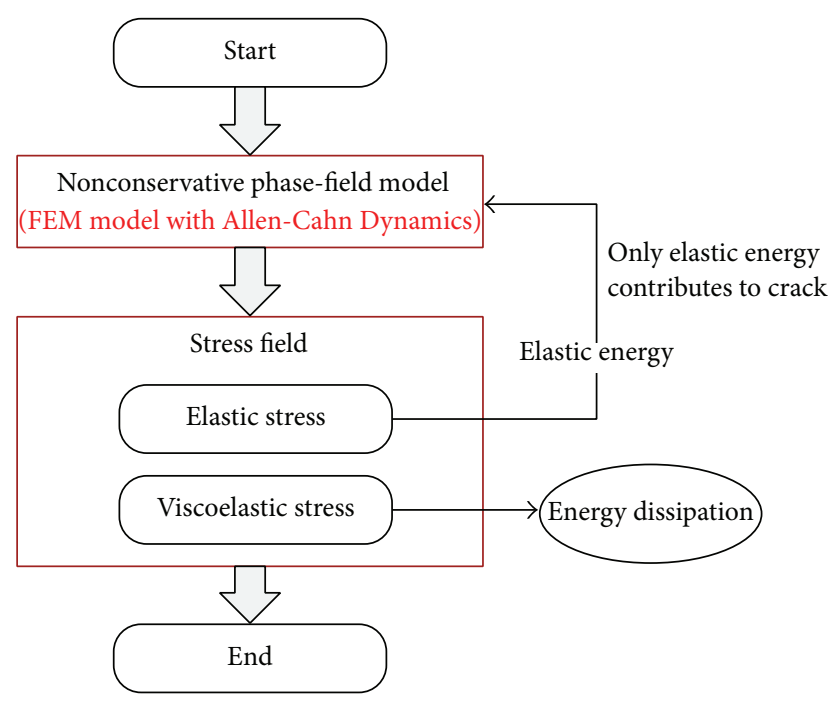

FIGURE 4: Quasi-brittle cracking calculation flowchart.

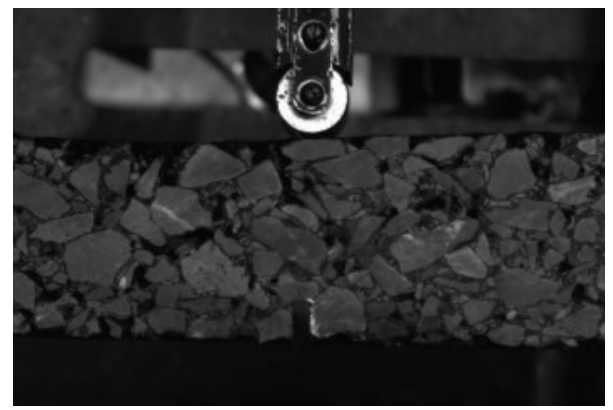

FIGURE 5: Asphalt mixture with coarse aggregates.

where $D$ corresponds to the kinetic coefficient and $\psi$ is the chemical potential:

$$
\psi=\frac{\delta F}{\delta \phi}=-\nabla \cdot \lambda \nabla \phi+\frac{\lambda}{\epsilon^{2}}\left(\phi^{2}-1\right) \phi+\frac{\partial f_{\mathrm{el}}}{\partial \phi} .
$$

Figure 4 shows the whole computation scheme.

\section{Results}

3.1. Applicability. The derivations of our previous theoretical analysis based on PFM lies in that bituminous material can be considered isotropic and homogenous. However, in reality, there will exist large coarse aggregates in asphalt mixture, as shown in Figure 5. In this case, the accuracy of PFM will be reduced. Nevertheless, if the asphalt concrete could be approximately considered as isotropic and the crack goes through the bitumen part other than through the aggregate, the PFM theory can still be used for approximation.

The cracking simulation using the diffuse interface approach used in this paper could be not only good for preflawed bitumen quasi-brittle cracking but also good for bituminous mixture using fine aggregates (Figure 6(a)). This method will not have high accuracy for bituminous mixture using coarse aggregates (Figure 6(b)).
3.2. Theoretical Stress Analysis. The authors have previously analyzed the asphalt binder cracking using phase-field method at low temperature [15]. However, there still have been two limits: (1) the previous research is focused on the asphalt linear behavior and does not consider the quasi-brittle behavior; (2) there lacks analytical solutions on the stress during cracking. To solve the second problem, the following equations are derived.

Consider the preflaw in bitumen specimen as a rectangular crack with depth $w$ and width $d$. The analytical expressions for stress are first obtained. For brittle cracking, Akono et al. (2011) suggested the specific $J$-integral expression for a rectangular crack propagation as [18]

$$
J=\frac{1}{p} \int_{A}\left(W n_{x}-t_{i} \frac{\partial u_{i}}{\partial x}\right) d A,
$$

where $p=w+2 d$ is the perimeter edge of the crack. $w$ is the crack width and $d$ is crack depth. Figure 7 shows the cracking contour. Note that in our research $\sigma_{x x}=0$, and all of the elastic strain energy is contributed by $\sigma_{y y}$ which is different from Akono et al.s previous work [18].

Based on Hooke's law, we further have elastic strain energy density as $W=(\kappa / 2 E) \sigma_{y y}^{2} ; d A=(w+2 d) d s$, and $d s=w d z$. Since the $J$-integral only has physical meaning when crack begins to propagate, we substitute the critical value $F=F_{0}$ into (11) and we have $\sigma_{y y}=F_{0} /\left(S-l_{0} d\right)$.

Since there is no traction on the fracture surface, we thus have

$$
J=\frac{1}{w+2 d} \int_{0}^{d} W w d z=\frac{\kappa w d \sigma_{y y}^{2}}{2 E(w+2 d)}
$$

where $\kappa=1-v^{2}$ for plane stress and $\kappa=1$ for plane strain.

Also, note that, during the linear elastic cracking process, the elastic strain energy will be totally transformed to the fracture energy $G$; that is,

$$
J=G=\frac{\kappa K_{C}^{2}}{E} .
$$

Compare (11) and (12), we now have

$$
\begin{aligned}
& \sigma_{y y}=\sqrt{2} K_{C} \sqrt{\frac{1}{d}+\frac{2}{w}}, \\
& \sigma_{y y}=k \sigma=\frac{k F}{S},
\end{aligned}
$$

where $k$ is the stress concentration factor due to the existence of visco- and plastic properties in bitumen.

Equation (14) indicates the relationship between the premade crack dimensions and stress based on the assumption of linear fracture mechanics, which needs to be further modified if the bitumen cracking is not quasi-brittle. It is observed that, for a given $K_{C}$, the smaller the initial crack is, the larger the stress is.

3.3. Numerical Simulations. A two-dimensional finite element model is established in COMSOL to simulate the quasibrittle cracking process of bitumen under tension loading. 


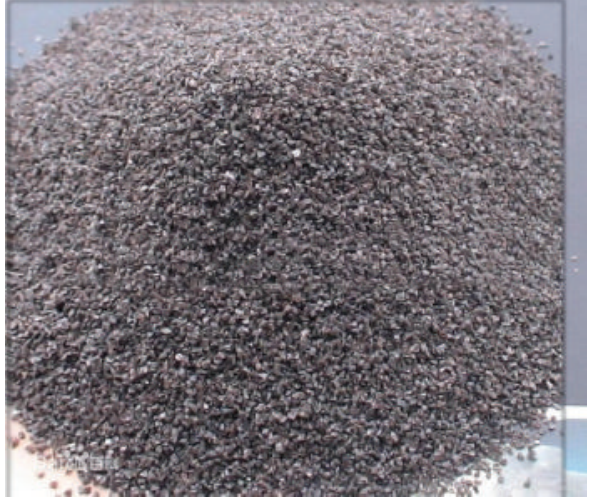

(a)

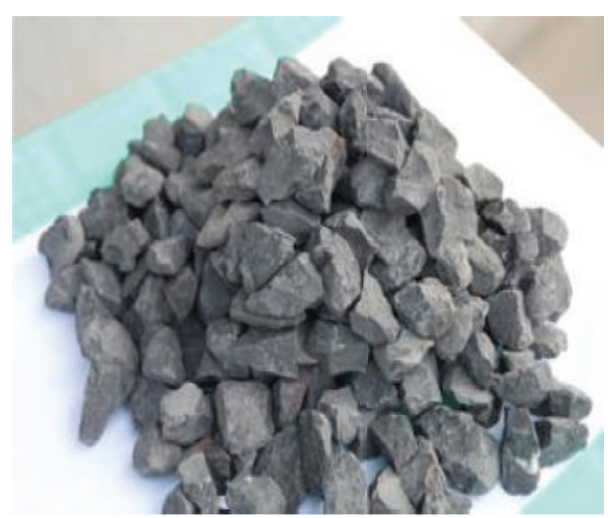

(b)

FIGURE 6: (a) Fine aggregates and (b) coarse aggregates.

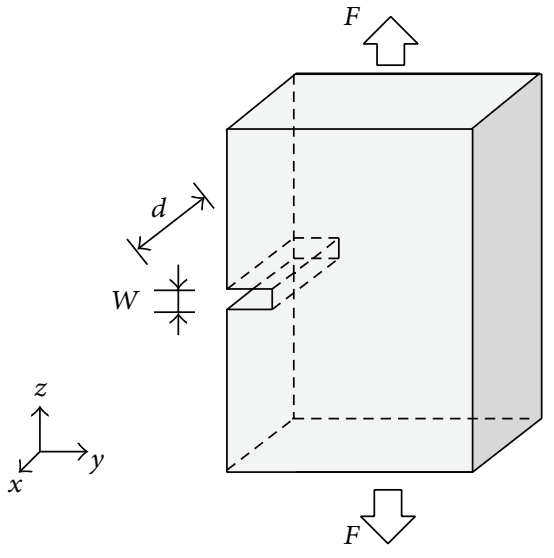

Figure 7: Cracking contour.

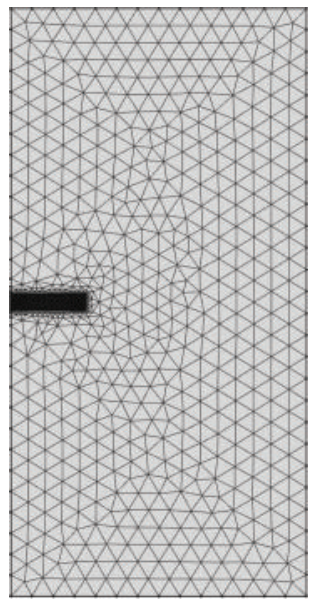

FIGURE 8: Fixed Eulerian mesh in preflawed bitumen model.

A fixed Eulerian mesh is used to describe the internal interfaces between the intact solid and crack void, as shown in Figure 8.

Actually the overall cracking process in preflawed bitumen should be simulated in three dimensions. However, it will be very costly to do such simulation. Previous researches
$[10,19]$ show that two-dimensional simplification, that is, plain strain or plain stress, will have sufficient accuracy for small size bitumen specimen. In our case, consider that the strain on the $z$ direction almost remains the same during cracking, and the plain strain assumption is used for simplification and the computational domain is a rectangular with dimensions $0.04 \times 0.02 \mathrm{~m}$. The thickness of the preflawed bitumen model is set as $0.01 \mathrm{~m}$. The interfacial thickness is $\epsilon=$ $0.001 \mathrm{~m}$ and the initial crack length is $a=0.005 \mathrm{~m}$. The finite crack width is $W=0.001 \mathrm{~m}$. The six-node triangular elements (P2 element) are used in the finite element calculations. 78,061 elements are used in the initial mesh. To solve the phase-field variable $\phi$ across the interface conveniently, the self-adaptive meshing is employed. The whole computation is conducted on a PC with Intel Core i7-4702HQ CPU, which takes about 2 hours computational time. It is expected that the smaller the interface coefficient is and the more the meshing is used, the more accurate the results will be.

Pure tension loading is applied on top and bottom boundaries, which is set as $\sigma(t)=\left(\sigma_{0} / t_{0}\right) \cdot t$ where $\sigma_{0} / t_{0}=$ $2 \times 10^{6} \mathrm{~N} / \mathrm{m}^{2} \mathrm{~s}$. The time step $\Delta t$ is set as $0.1 \mathrm{~s}$, considering our computational ability.

To study the quasi-brittle cracking behavior and viscoelastic cracking behavior on bitumen, two simulations are conducted simultaneously. The first is to consider the bitumen as linear elastic, while the second is to consider bitumen as viscoelastic plastic.

Case 1. Quasi-brittle cracking: the following material properties are used: $\gamma=183.77 \mathrm{~J} / \mathrm{m}^{2}, E=5.8 \mathrm{MPa}$, and Poisson's ratio $v=0.3$.

Case 2. Viscoelastic cracking is used to reflect the viscoelastic property of bitumen.

In order to study the differences between quasi-brittle cracking and viscoelastic cracking in our phase-field model on bitumen, the fracture results are first calculated and compared to classic fracture mechanics, where the Griffith criterion is $K_{I} \geq K_{I C}, K_{I}$ is the mode $I$ stress intensity factor and $K_{I C}$ is the mode $I$ fracture toughness which is a material parameter. 
For bitumen at low temperature, $K_{I C}=48 \mathrm{kNm}^{-3 / 2}$ by Ponniah et al. [20] is selected as facture toughness. The elastic modulus of bitumen specimen is $5.8 \mathrm{MPa}$, which corresponds to a fracture energy $G_{C}=368 \mathrm{~J} / \mathrm{m}^{2}$.

The critical load calculation based on the classic fracture mechanics without considering viscous property can be calculated as $313310.96 \mathrm{~Pa}$. In phase-field method, the load value at the time instant when $\phi$ reaches -1 is considered to be the critical load.

For Case 1, where bitumen brittle-cracking occurs, our simulation results show that at time step $=16 \Delta t$ critical load happens, which corresponds to a load as $320000 \mathrm{~Pa}$.

For Case 2, where bitumen brittle-cracking occurs, our simulation results show that at time step $=31 \Delta t$ critical load happens, which corresponds to a load as $620000 \mathrm{~Pa}$.

The reason why the critical load in quasi-brittle phasefield model is much smaller than that in the viscoelastic model is that only the elastic stress part will contribute to fracture rather than the total stress (including elastic stress, viscous stress, and plastic stress) during brittle-cracking. It means bitumen has more resistance to fracture when considering viscoelastic properties than pure brittle linear elastic.

For infinitesimal deformation during bitumen brittle cracking, Cauchy stress is the same as Piola-Kirchhoff stress, which indicates the pure brittle cracking. However, considering the "quasi-brittle" property, finite deformations will occur at crack tip, and the second Piola-Kirchhoff stress is used to express the stress with Gauss-point evaluation. For the $x$ component of second Piola-Kirchhoff stress at $t=1.6 \mathrm{~s}$ at the crack tip, the calculation result is $1.7 \times 10^{6} \mathrm{pa}$, which is very large compared with other regions.

\section{Summary}

In this paper, we present a phase-field model to analyze the quasi-brittle fracture in bitumen subject to tension loading. In phase-field model, only the elastic stress will contribute to the fracture. Overall, compared with the classic fracture mechanics, the phase-field model does not need to explicitly treat the crack surface and can easily solve the quasi-brittle cracking of preflawed bitumen specimen.

\section{Competing Interests}

The authors declare that they have no competing interests.

\section{Acknowledgments}

The research performed in this paper is supported by National Natural Science Foundation of China (no. 51308042 and no. 41372320), Natural Science Foundation of Shandong Province (ZR2015EQ009), and the Fundamental Research Funds for the Central Universities (06500036).

\section{References}

[1] C. Peterson, W. Buttlar, and A. Braham, "Mixed-mode cracking in asphaltic concrete," Advanced Testing and Characterization of Bituminous Materials, vol. 2, pp. 785-795, 2009.

[2] E. V. Dave and W. G. Buttlar, "Thermal reflective cracking of asphalt concrete overlays," International Journal of Pavement Engineering, vol. 11, no. 6, pp. 477-488, 2010.

[3] D. A. Anderson, D. W. Christensen, H. U. Bahia, M. G. Sharma, C. E. Antle, and J. Button, "Binder characterization and evaluation," Strategic Highway Research Program (SHRP) A-369, National Research Council, 1994.

[4] M.-R. Ayatollahi and S. Pirmohammad, "Temperature effects on brittle fracture in cracked asphalt concretes," Structural Engineering and Mechanics, vol. 45, no. 1, pp. 19-32, 2013.

[5] G. M. Rowe, G. King, and M. Anderson, "The influence of binder rheology on the cracking of asphalt mixes in airport and highway projects," Journal of Testing and Evaluation, vol. 42, no. 5, pp. 1063-1072, 2014.

[6] E. V. Dave, S. H. Song, W. G. Buttlar, and G. H. Paulino, "Reflective and thermal cracking modeling of asphaltic concrete overlays," Advanced Testing and Characterization of Bituminous Materials, vol. 2, pp. 1241-1252, 2007.

[7] B. Birgisson, B. Sangpetngam, and R. Roque, "Predicting viscoelastic response and crack growth in asphalt mixtures with the boundary element method," Transportation Research Record, vol. 1789, pp. 129-135, 2002.

[8] M. Ameri, A. Mansourian, M. Khavas, M. Aliha, and M. Ayatollahi, "Cracked asphalt pavement under traffic loading-a 3D finite element analysis," Engineering Fracture Mechanics, vol. 78, no. 8, pp. 1817-1826, 2011.

[9] Y. Tan and M. Guo, "Interfacial thickness and interaction between asphalt and mineral fillers," Materials and Structures, vol. 47, no. 4, pp. 605-614, 2014.

[10] Y. Hou, P. Yue, Q. Xin, T. Pauli, W. Sun, and L. Wang, "Fracture failure of asphalt binder in mixed mode (Modes I and II) by using phase-field model," Road Materials and Pavement Design, vol. 15, no. 1, pp. 167-181, 2014.

[11] X.-J. Feng, P.-W. Hao, and X.-D. Zha, "Research on low temperature anti-cracking performance of the ATB30 asphalt treated base," Journal of Wuhan University of Technology, vol. 29, no. 6, pp. 35-49, 2007.

[12] S. Mun and H.-J. Lee, "Modeling viscoelastic crack growth in hot-mix asphalt concrete mixtures using a disk-shaped compact tension test," Journal of Engineering Mechanics, vol. 137, no. 6, pp. 431-438, 2011.

[13] Y. Zhao and D. Zhang, "Study of low temperature cracking of asphalt pavement based on cohesive zone model," Journal of Highway and Transportation Research and Development, vol. 27, no. 1, pp. 11-16, 2010.

[14] A. A. Griffith, "The phenomena of rupture and flow in solids," Philosophical Transactions of the Royal Society of London A, vol. 221, no. 582-593, pp. 163-198, 1921.

[15] Y. Hou, L. Wang, P. Yue, T. Pauli, and W. Sun, "Modeling mode I cracking failure in asphalt binder by using nonconserved phasefield model," Journal of Materials in Civil Engineering, vol. 26, no. 4, pp. 684-691, 2014.

[16] J. W. Cahn and J. E. Hilliard, "Free energy of a nonuniform system. I. Interfacial free energy," The Journal of Chemical Physics, vol. 28, no. 2, pp. 258-267, 1958. 
[17] M. Fleck, Solid-state transformations and crack propagation: a phase field study [Ph.D. thesis], University of Bayreuth, Bayreuth, Germany, 2011.

[18] A.-T. Akono, P. M. Reis, and F.-J. Ulm, "Scratching as a fracture process: from butter to steel," Physical Review Letters, vol. 106, no. 20, Article ID 204302, 2011.

[19] D. Gross and T. Seelig, Fracture Mechanics: With an Introduction to Micromechanics, Springer, New York, NY, USA, 2006.

[20] J. E. Ponniah, R. A. Cullen, and S. A. Hesp, "Fracture energy specifications for modified asphalts," Proceedings in ACS Division of Fuel Chemistry, vol. 41, no. 4, pp. 1317-1321, 1996. 

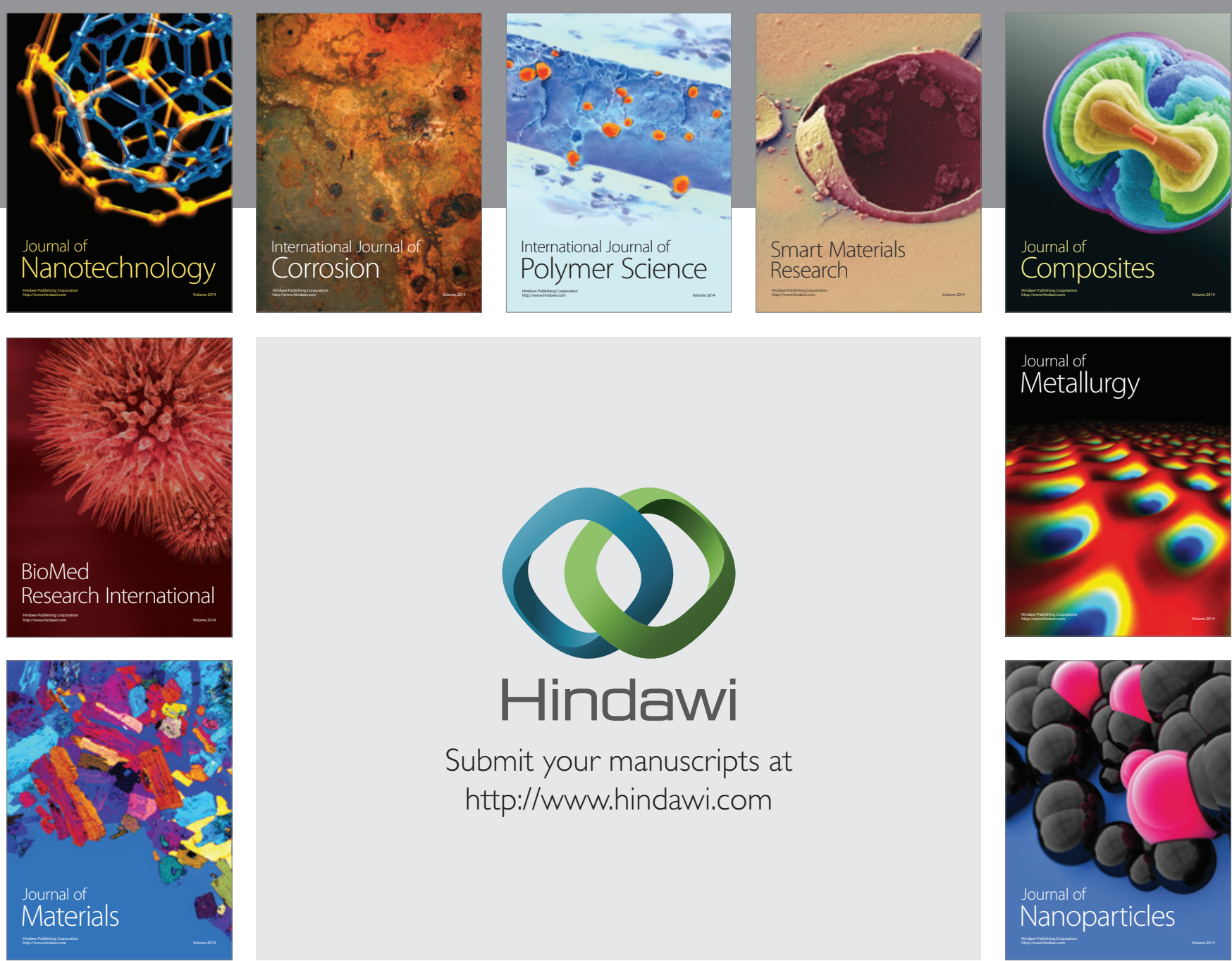

\section{Hindawi}

Submit your manuscripts at

http://www.hindawi.com

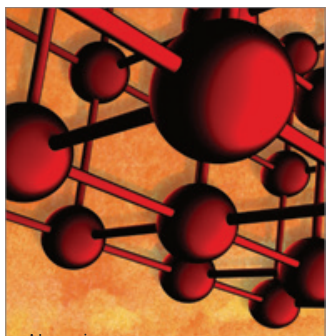

Materials Science and Engineering
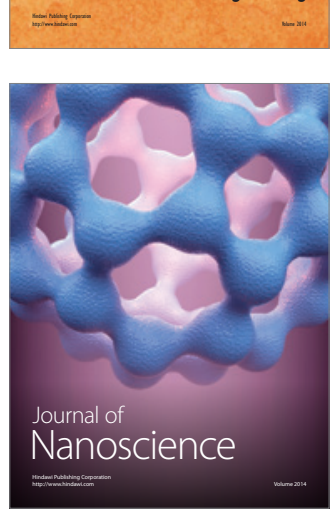
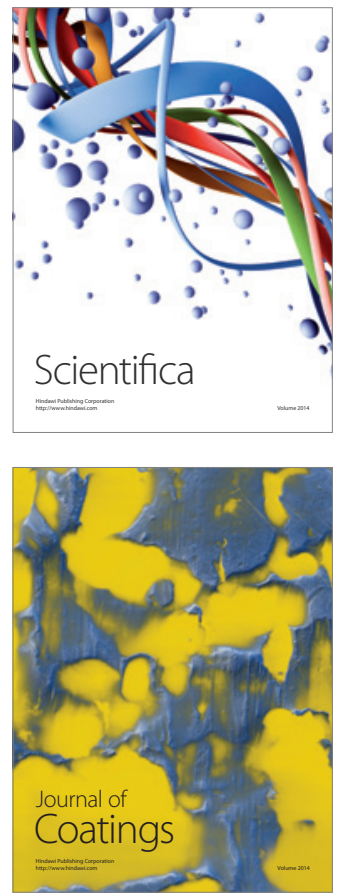
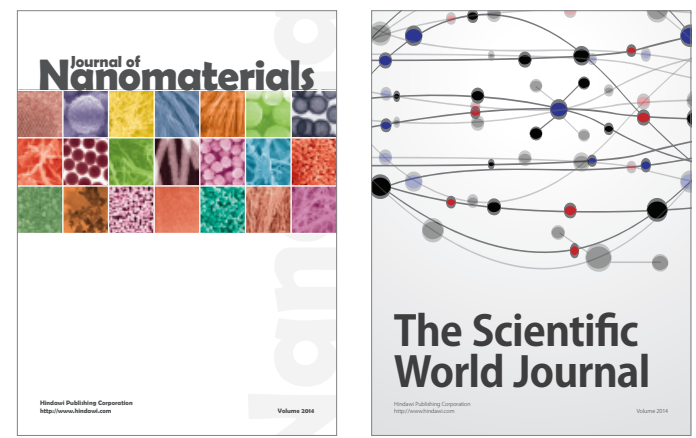

The Scientific World Journal
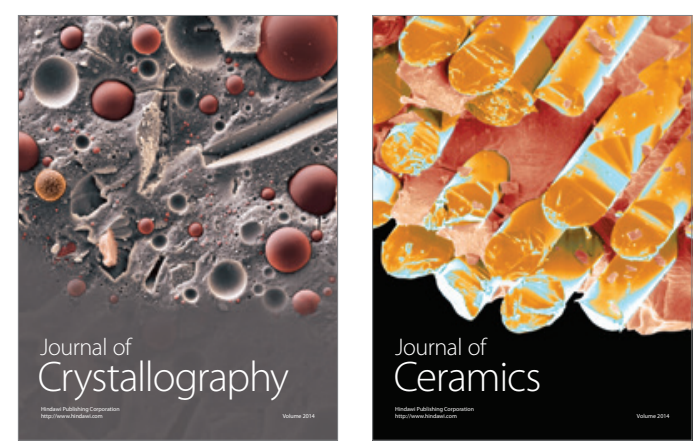
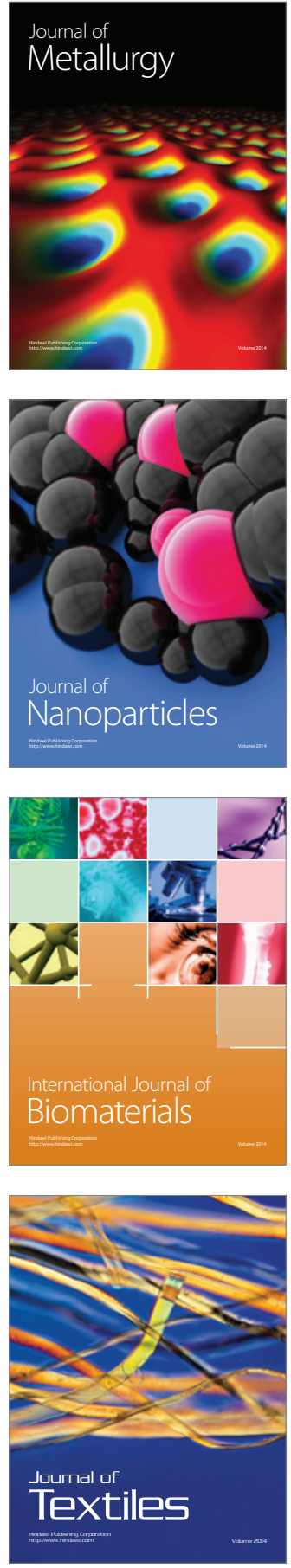